\title{
Could the pandemic affect sexually-transmitted disease incidence in high-risk groups? Considerations about the COVID-19 lockdown
}

\author{
A pandemia pode afetar a incidência de doenças \\ sexualmente transmissíveis em grupos de \\ alto risco? Considerações sobre o \\ isolamento social na COVID-19
}

\author{
¿Podría la pandemia afectar la incidencia de \\ enfermedades de transmisión sexual en grupos \\ de alto riesgo? Consideraciones sobre el \\ confinamiento durante la COVID-19
}

Rocco Salvatore Calabrò 1

Giuseppe Gervasi 2

doi: 10.1590/0102-311X00009521

Dear Editors,

We read with great interest the work by Sousa et al. ${ }^{1}$ on factors associated with sex practice under the effect of drugs among men who have sex with men (MSM) during the period of social isolation in the context of the COVID-19 pandemic. In their multicentric online survey, the authors found that a significant portion (95\%) of MSM that engaged in chemsex did so in sex with casual partners during the pandemic's upward epidemiological curve in both countries (April 2020). Moreover, chemsex practice in Brazil exceeded that in Portugal by more than twice.

The outbreak of coronavirus disease 2019 (COVID-19) tremendously impacted healthcare and economic systems worldwide. COVID-19 is an acute respiratory illness caused by the SARS-CoV-2. The high susceptibility of the general population to this virus' contagion led to an overall negative impact on personal and social relationships, since transmission occurs via droplets or direct contact with contaminated objects (fomites).

As such, the first-line preventive strategies are to increase social distancing, and rise up all the general prevention measures focusing on the infective respiratory diseases (i.e., droplet barriers and contact isolation) 2 . These important personal limitations, along with the huge negative impact caused by media and social networks, led to a growing fear of close relationships. The condition of personal isolation is more evident when facing the reduction of fortuitous sexual intercourse, as reported by recent surveys in general population 3,4, in contrast with what Sousa et al. ${ }^{1}$ found in the MSM group.

It is noteworthy that some authors described the presence of SARS-CoV-2 in feces, as well as in the seminal fluids of male subjects, including those who did not present symptoms and signs of COVID-19. These findings could lead to a potential role of sexual transmission in this disease.

Among the categories at higher risk of STIs, MSM might represent an important public health challenge during the COVID-19 pandemic 5. MSM is a particular risk group of STIs, requiring specific health interventions. MSM is, in fact, the group with the highest prevelance of HIV contagions and subsequently AIDS. In particular, the limited access to the first-line preventive measures (i.e., condoms) in some countries, along with their high rate of substances of abuse seem to explain the high rates of unprotected anal intercourse (up to 84\%) in MSM 5. Moreover, the experience of community discrimination, with social stigma and subsequent isolation, determined the growing of unorthodox sexual behaviors, with an increased risk of STIs. In addition, the fear of social discrimi-

1 IRCCS Centro Neurolesi Bonino-Pulejo, Messina, Italy.

2 Department of Prevention, Local Health Authority of Viterbo, Rome, Italy.

Correspondence R. S. Calabrò IRCCS Centro Neurolesi Bonino-Pulejo. Via Palermo, Cda Casazza SS113, Messina 98122 Sicily, Italy. salbro77@tiscali.it 
nation could negatively affect vaccination for STIs in MSM, as the group shows low compliance to vaccination schedules 5,6.

In the context of COVID-19 pandemic, the management of STI risk for MSM could be very complex. The fear for a potentially fatal disease, which can cause death in a short period of time, might determine the avoidance of the sexual activities requiring a close contact with the partner, such as kissing or soft sex. However, the experienced indifference to STI risk, including HIV, could lead to the increase of unprotected fast sex through oral and, especially, anal intercourse 7 . Moreover, as suggested by the authors, during the prolonged "quarantine", moments of casual sex with anonymous partners under the effect of drugs and with multiple partners may be interpreted as a brief moment of "relaxation" and escape from reality ${ }^{1}$. The maintenance of this practice at high levels during the pandemic raises concern, potentially reinforcing the thought that social isolation was not effective in Brazil, especially among MSM.

Thus, a tailored sexual intervention should be promoted to support these "fragile" groups, such as MSM, during the COVID-19 pandemic. In particular, a multidisciplinary approach integrating biomedical, behavioral and social perspectives, along with a specific educative intervention for the particular social background, could be helpful. Epidemiological studies on the prevalence of STIs during the COVID-19 pandemic should be also fostered to evaluate if and to which extent sexually risky behaviors decrease during pandemics.

\section{Contributors}

The authors equally contributed to the work.

\section{Additional informations}

ORCID: Rocco Salvatore Calabrò (0000-00028566-3166); Giuseppe Gervasi (0000-0001-8527645X).

\section{Conflict of interests}

The authors declare no conflict of interests.
1. Sousa AFL, Queiroz AAFLN, Lima SVMA, Almeida PD, Oliveira LB, Chone JS, et al. Chemsex practice among men who have sex with men (MSM) during social isolation from COVID-19: multicentric online survey. Cad Saúde Pública 2020; 36:e00202420.

2. Fisher D, Heymann D. Q\&A: the novel coronavirus outbreak causing COVID-19. BMC Med 2020; 18:57.

3. Li W, Li G, Xin C, Wang Y, Yang S. Challenges in the practice of sexual medicine in the time of COVID-19 in China. J Sex Med 2020; 17:1225-8.

4. Jacob L, Smith L, Butler L, Barnett Y, Grabovac I, McDermott D, et al. Challenges in the practice of sexual medicine in the time of COVID-19 in the United Kingdom. J Sex Med 2020; 17:1229-36.

5. de Vries HJC. Sexually transmitted infections in men who have sex with men. Clin Dermatol 2014; 32:181-8.

6. Calabrò RS, Quattrini F. Disclosing sexual orientation to healthcare providers: still a taboo or time for a change? J Natl Med Assoc 2018; 110:596-7.

7. Cipriano M, Giacalone A, Ruberti E. Sexual behaviors during COVID-19: the potential risk of transmission. Arch Sex Behav 2020; 49:1431-2.

Submitted on 14/Jan/2021

Approved on 22/Jan/2021 